\title{
A User-Oriented Adaptive-Optimal Car Parking Management System Towards Smart Livings
}

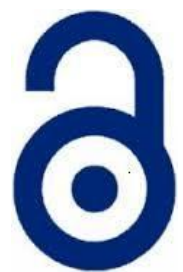 \\ Received: 17 April 2021 \\ Accepted: 12 May 2021 \\ Published: 15 July 2020 \\ Publisher: Deer Hill Publications \\ (c) 2021 The Author(s) \\ Creative Commons: CC BY 4.0
}

Sato Taiga, A. S. M. Bakibillah, Kotaro Hashikura, Md Abdus Samad Kamal and Kou Yamada

\begin{abstract}
Existing parking management approaches do not consider specific requirements, priorities, user comfort, or modes of use when allocating a parking spot in a large park. As a result, vehicles carrying multiple passengers but staying for a limited period often have to drive further, searching for a parking spot, which increases fuel consumption, emissions, waste of time, and discomfort of users due to extra walking distance. In this paper, we consider the need for both sustainability and comfortable livings in a future smart city and propose an adaptive-optimal scheme that takes advantage of parking efficiency based on the passenger information and flexibly provides the optimal parking spot to the individual. We presume that the management system has information about the number of users, user priority, and expected stay time when a car arrives or a parking request is made. The best parking slot is assigned based on the available parking slots and the given objectives, such as the shortest travel distance inside the parking zone for a low pollution, the shortest walking distance per user, or a combination of both with some trade-off. The decision process is fine-tuned using parking data obtained from a model of a large car park of a shopping complex, and the results of the proposed scheme are compared with other schemes. The findings indicate that overall time spent in the parking lot, as well as individual walking and travel distances, have significantly improved.
\end{abstract}

Keywords: Smart car parking, Cloud computing, Adaptive optimization, Dynamic parking allocation, Smart livings.

\section{INTRODUCTION}

In the last few decades, private cars have been the most popular mode of urban transportation. The number of cars in the world is projected to rise significantly from 841 million in 2008 to over 1.6 billion in 2035 [1]. The growing number of personal cars on the road leads to increased traffic congestion, fuel consumption, emissions, and time wastage. In addition, drivers looking for parking spaces account for about 30\% of total traffic [2]. According to a global parking survey conducted by IBM in 2011, the average time spent looking for a desirable parking spot is 20 minutes [3]. In most modern cities, finding unoccupied parking spaces is a common issue as individuals arrive by car and need to visually check for vacant spaces, resulting in a large number of vehicles competing for a few available parking spaces, which causes traffic congestion in parking lots. In such situations, it becomes more difficult for vehicles to locate a vacant parking lot in a timely manner, and they are often unable to pass, even though they wish to leave the congested area. Specifically, large parking lots, such as those found in shopping centres, hospitals, airports, and stadiums, are sources of time-wasting, fuel consumption, and pollution, all of which have an effect on individual users and their social lives. One of the major problems is the mismanagement of available parking spaces, which makes it difficult for users to find a parking spot in a timely manner. Therefore, it is highly desirable to develop a smart car parking management system that considers individual needs and usage while providing optimal services. The primary objective of a smart car parking management system is to identify, assign, and reserve the optimal accessible car parking lot for a user driving in a specific location.

In recent years, the idea of a smart city for smart livings has gained popularity in many countries. Car parking facilities and traffic management systems are two of the most important concerns in smart cities [4-5], which can be resolved by Intelligent Transport Systems (ITS). Recent advancements in inter-vehicle communication, Internet of Things (loT), and sensing technologies, which share information between vehicles and roads, have allowed for improvements in parking lot navigation performance, such as comprehensive entry route guidance in large parking lots and driving route guidance before entering the parking lot [6]. Inline, the cloud is an ideal partner for loT because

S. Taiga', A. S. M. Bakibillah², K. Hashikura, M. A. S. Kamal'凶 and K. Yamada 'Graduate School of Science and Technology, Gunma University, Kiryu, Japan

${ }^{2} S c h o o l$ of Engineering, Monash University, Bandar Sunway, Malaysia

E-mail: maskamal@gunma-u.ac.jp

Reference: Taiga et al. (2021). A User-Oriented Adaptive-Optimal Car Parking Management System Towards Smart Livings. International Journal of Engineering Materials and Manufacture, 6(3), 202-208. 
it serves as a platform for storing and accessing sensor data from remote locations [7]. If parking information is given to drivers or cars can be dispatched automatically by interacting with them, these efforts are supposed to save a lot of time, driving distance, and walking distance.

For the future smart society, various loT-based car parking systems have been proposed in the literature. In [8], an loT-based cloud-integrated smart parking system is proposed, which includes installing an loT module on-site, and tracking and communicating each parking space's availability. In [9], a cloud-based intelligent car paring service is developed for smart cities, which consists of sensors, communication systems, and devices. In [10], an automated thresholding algorithm-based approach for detecting car parking lots is proposed. Due to the high cost of image processing algorithms, a hardware approach is developed. Another research developed an innovative smart carparking system based on intelligent resource allocation, reservation, and pricing [11]. The new system is based on mathematical modelling using mixed-integer linear programming (MILP), with the goal of lowering overall monetary costs for drivers while increasing parking resource usage. A multiagent agent system based on InfoStation is proposed to facilitate a car parking locator service [12]; users are provided with customized services based on their location and mobile device capabilities. Some researchers suggested a new scheme that included a parking assistance service [13]. The assigned parking space will be shown on a small map using wireless transmission for cars using the dedicated short-range communication protocol DSRC when entering the car park. The car is directed to the allocated space using an inertial navigation system (INS). Some other work proposed a multi-layer architecture for smart parking systems consisting of multi-parametric parking slot sensor nodes, the latest long-range low-power wireless communication technology, and Edge-Cloud computation [14]. The developed scheme facilitates dynamic parking management for broad areas via near real-time vehicle tracking, while providing valuable information to drivers about available parking spaces and related services.

The above works did not consider individual needs, preferences, user convenience, or modes of use when allocating a parking spot in a large park. Hence, vehicles carrying many passengers but staying for a limited period of time often have to drive further in search of a parking spot. Consequently, parking increases fuel consumption and pollution, as well as the additional walking distance per person. In this paper, we recognize the need for both sustainability and comfortable living in a future smart city, and we propose a scheme that takes advantage of parking efficiency based on passenger information and flexibly provides the person with the best parking spot. We assume that when a car arrives or a parking request is made, the management system has details about the number of users, their priority, and the duration of their expected stay. Based on the available parking slots and the given goals, the best parking slot is allocated. The findings indicate a substantial reduction in overall fuel consumption and person walking distance in the parking lot.

The rest of the paper is organized as follows. Section II presents the proposed smart car parking management system, including the concept and system architecture, modelling of smart car parking, schemes for parking allocation, and car parking simulation model. Section III describes the simulation results. Section IV concludes the paper.

\section{SMART CAR PARKING MANAGEMENT SYSTEM}

\subsection{Concept and System Architecture}

Figure 1 illustrates the basic concept and architecture of the proposed smart car parking management system. The parking lot management system includes a system for interacting with cars as well as sensors for monitoring parking lot conditions. In order to support vehicles that do not have a communication facility, a camera for image processing and a display for guidance are installed. In this manner, the status of the parking lot is always updated on the cloud. Before a car enters the parking lot from the road, the number of people in the car, the intent of their visit, the time of stay, and other information is sent to the cloud-based management system, which utilizes the information to decide optimal car parking in real-time. Note that the implementation of an adaptive optimization scheme, as described in the next section, is the most important aspect of the architecture. After computation, the car near the entrance is given the ID of the parking space through communication or display. The status of the parking space is transmitted to the management system via sensors when a car enters and parks in the parking lot. 


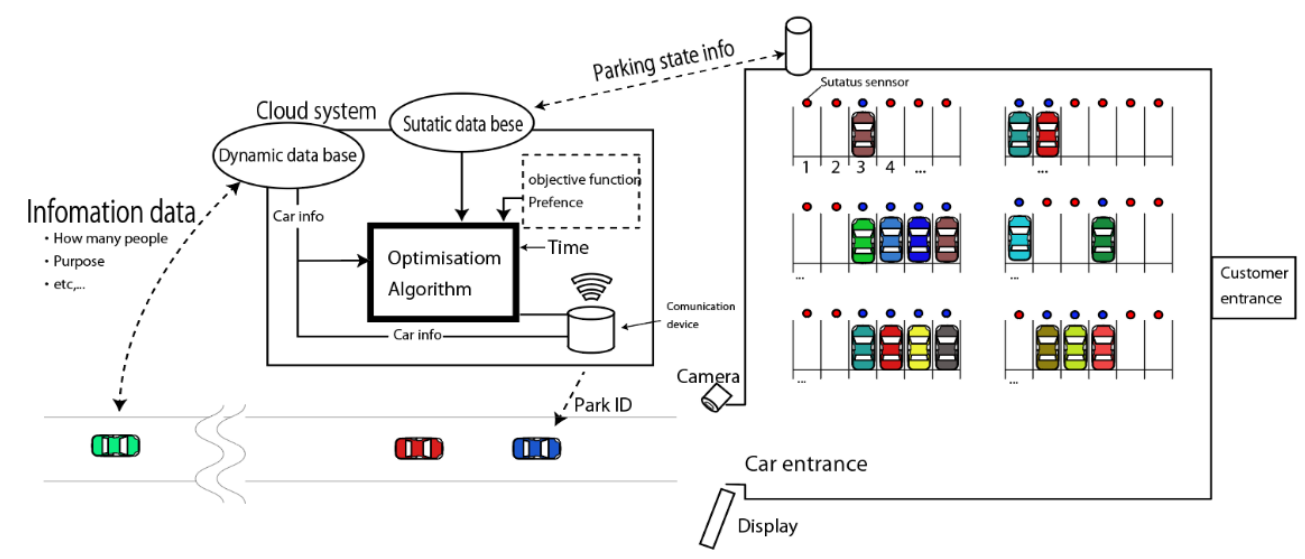

Figure 1: Concept and architecture of the proposed smart car parking management system.

\subsection{Modelling of Smart Car Parking}

For the necessity of modelling the smart car parking system, we consider the parking space has a capacity of $N_{P}$. For each parking slot $p=1,2, \ldots \ldots, N_{P}$, the optimal driving distance $d_{P}(p)$ between the car entrance and parking point, and the optimal exit distance $d_{E}(p)$ from the parking point to the exit are known. The walking distance $d_{W a l k}(p)$ from the parking point to the shopping mall entrance is also known. Such information is stored in a static database of the proposed cloud-based management system. Note that from the parking lot, there could be several customer entrances to the shopping center. However, we believe that people will always walk to and from their cars along the best walkway, e.g., minimum distance. On the other hand, the necessary information of each parking at time $t$ are stored in a dynamic database, which includes the status of the parking point given by a binary variable $\delta_{P}(t, p) \in$ $\{0,1\}$, where 0 denotes empty and 1 denotes occupied, and the remaining expected occupancy time $\tau_{E}\{t, p\}$ by the car.

Customers usually choose to park their car near the shop entrance to reduce the amount of time they have to walk and the pressure of carrying their belongings. This could be one of the objectives of the proposed system. However, assigning all cars to be parked near the shopping entrance is not feasible, and such a strategy may often result in a hotspot of pollution. To minimize pollution in a closed parking area, it is better to park in a location that decreases the travel distance inside the parking area. Furthermore, cars arriving within a certain time frame should not be directed to the same parking area because they could take longer to complete the parking and waste more fuel. Allocating the best spot for a car that will be parked for a long time might not be the best option for a car that will be parked for a short period of time. It is important to find appropriate parking places for each car that provide the best overall service to all, taking into account vehicle arrival trends and parking occupancy. Thus, it is presumed that each car's stay period is approximately known.

Considering the above facts, preferences, and constraints, we minimize the following objective function by selecting the appropriate $p$ for each car $c$.

$$
J=\sum_{d=1}^{N_{\text {day }}} \sum_{c=1}^{N_{\text {cars }}} \alpha f_{\text {comfort }}(t, c, p)+(1-\alpha) f_{\text {environment }}(t, c, p),
$$

where $\alpha$ is the tuning factor, $f_{\text {comfort }}$ is the cost related to the comfort of the user, and $f_{\text {environment }}$ is the cost related to environmental aspects. The factor $\alpha$ should be correctly selected by observation and fine-tuning of the parameters. In traditional optimization, factor $\alpha$ is usually kept constant for all cars. However, it is preferable to find a different value of $\alpha$ for each passenger, and hence, we choose an adaptive factor $\alpha$ for each car that varies depending on the car users, stay time, and occupancy trends of the parking lots. The costs $f_{\text {comfort }}$ and $f_{\text {environment }}$ are given as

$$
\begin{aligned}
& f_{\text {comfort }}=w_{\mathrm{c}} 2 d_{\text {Walk }}, \\
& f_{\text {environment }}=w_{e 1}\left(d_{P}+d_{E}\right)+w_{e 2} \rho(t)+w_{e 3},
\end{aligned}
$$

where $w_{\mathrm{c}}$ denotes the multiplying factor, $w_{e 1}, w_{e 2}$, and $w_{e 3}$ are constant weights, and $\rho(t)$ is the extra complexity depending on the occupancy rate of the park. The reduction of $f_{\text {comfort }}$ may result in the least amount of walking time or distance between the car and the shop entrance, which is highly desired by users. The reduction of $f_{\text {environment }}$ means that the car's engine runs for the shortest possible time, reducing emissions and fuel waste in the parking and 
contributing to clean air. Therefore, the above optimization problem can be tuned for different objectives of parking management by choosing suitable $\alpha$.

\subsection{Schemes for Parking Allocation}

For the parking allocation task, we consider several schemes with different objectives as follows.

- Uncontrolled scheme: As one of the baseline schemes, a parking point is randomly selected by each car. The cars are distributed at random among the available parking spaces. This approach is used as the foundation for evaluating the rest of the schemes based on the above optimization problem.

- Least-walk scheme: The system looks for available parking spaces near the store's entrance. This can be considered a greedy selection scheme of parking points by the users in an uncontrolled scenario. The same results can be obtained from (1) if $\alpha$ is set to 1, i.e., the user's comfort is the only factor considered in the objective function.

- Least-travel scheme: All cars are looking for a parking spot in an available parking lot that is near to the car's entrance/exit and dispatched without regard for the user's comfort, i.e., $\alpha$ is set to 0 in the objective function. Since the total travel distance by a car in the parking lot can be minimum, it is also called a Minimum emission Parking scheme.

- Optimal scheme: With the trade-off for user comforts and emission reduction, the objective function is taken at a balanced condition by setting $\alpha$ at 0.5 .

- Adaptive-optimal scheme: Under this scheme, the balancing factor $\alpha$ is tuned dynamically, considering several factors for each car. Specifically, the number of passengers of a car, intended stay time, and trend of the congestion are used to tune $\alpha$ for improving the user comforts, parking utilization beyond the optimal scheme in a conventional sense while keeping the environmental aspect the same. Particularly, in this scheme for each car according to its arrival context, $\alpha$ is tuned as

$$
\alpha=\left\{\begin{array}{cc}
0 & \text { for } \tau_{E}\{t, p\}>2 \mathrm{hr}, \text { and } \theta\left(t+\tau_{E}\right)=1 \\
\min (0.5+(\eta(c)-1) / 3,1) & \text { otherwise }
\end{array}\right.
$$

where $\eta(c)$ denotes the number of passengers in car $c$ and $\theta\left(t, \tau_{E}\right)$ denotes the trend of the congestion level with 1 means peak congestion (with above $85 \%$ occupancy) is expected within the stay time of the car. If the peak times of the user arrival are known from the past data, and covering that time when a vehicle intends to stay for more than $2 \mathrm{hr}$, then $\alpha$ is set to 0 according to (4). In the other cases, $\alpha$ varies from 0.5 to 1.0 depending on the number of passengers in the car. In this way, the proposed adaptive optimization scheme provides the least walking park to the cars with a higher number of passengers while avoids blocking the most favourable parking point by the long-staying cars.

\subsection{Car Parking Simulation Model}

We develop a two-dimensional model of a real parking lot, as shown in Figure 2. It refers to the fourth floor of a multilevel car park with four levels. The parking lot is approximately 150 meters long and 71 meters wide, with 248 parking spaces. Each parking space is assigned an ID and details about its location, including the shortest distance between the car and human entrances and exits. There is one entrance and exit for cars and two entrances and exits for people. The parking space is 6.0 meters long and 2.5 meters wide, with a road width of 7 meters. There are two types of roads: one-way and two-way, which is a reference to the design standards for parking spaces in Japan. Buildings and other structures are shown in orange.

Figure 3 shows the distributions of car parks for different walking distance and driving distances. We collected approximate parking usage data for several days from 7:00 am to 11:00 pm and created a trend model for parked cars, as shown in Figure 4. In the study area, the stores operate from 9:00 am to 11:00 pm. Customers and store employees are the two categories of consumers, and the amount of time spent in the store, the amount of time spent in the parking lot, and the number of cars vary. Figure 5 shows the histograms of cars with varying users and their stay time. Though most cars come with a single user, a significant number of cars have more than one passenger. The cars stay for several hours are usually belong to the shop employees or staffs. Most customer cars stay less than 1 hour, and it is expected that providing the best parking point to them could improve the overall usage of the parking lot. 


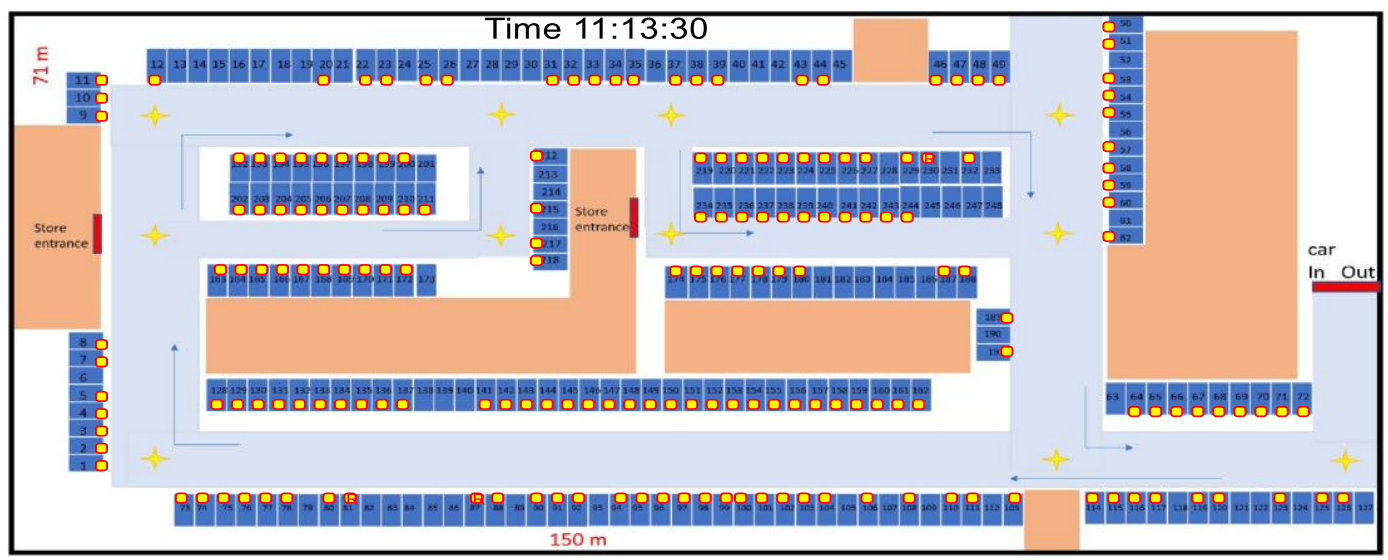

Figure 2: A simulation model using a real parking lot in Japan contains 248 parking points. The small yellow squares indicate an occupied parking point.

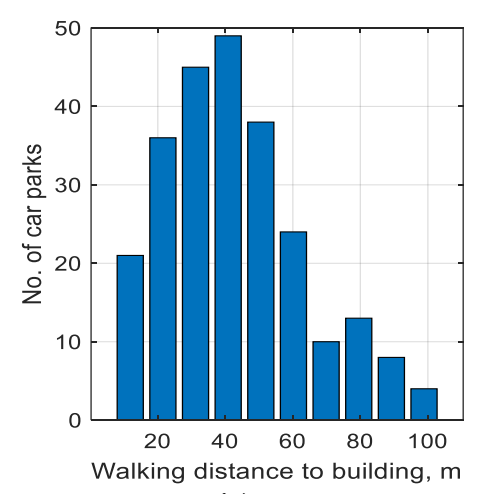

(a)

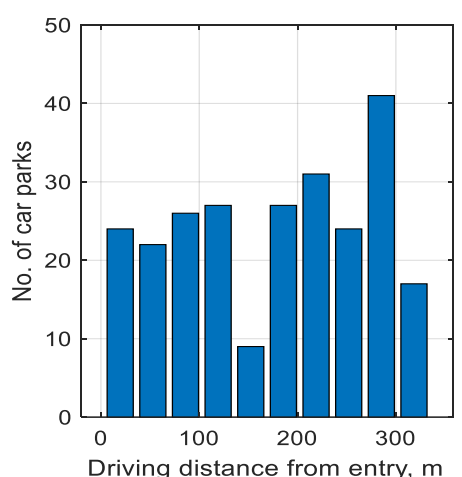

(b)

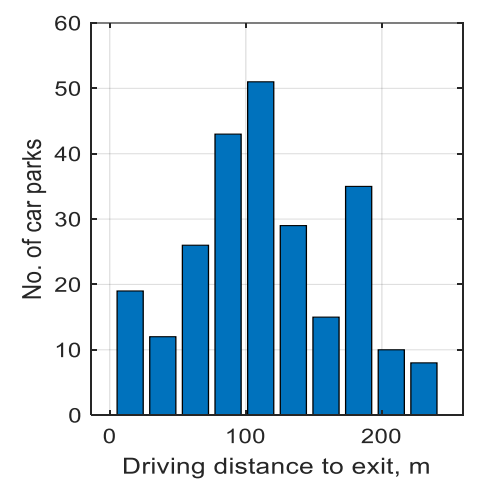

(c)

Figure 3: Histograms of car parks vs (a) walking distance from a parking point to the building entrance, (b) driving distance from the parking lot entrance to a parking point, and (c) driving distance from the parking point to the parking lot exit.

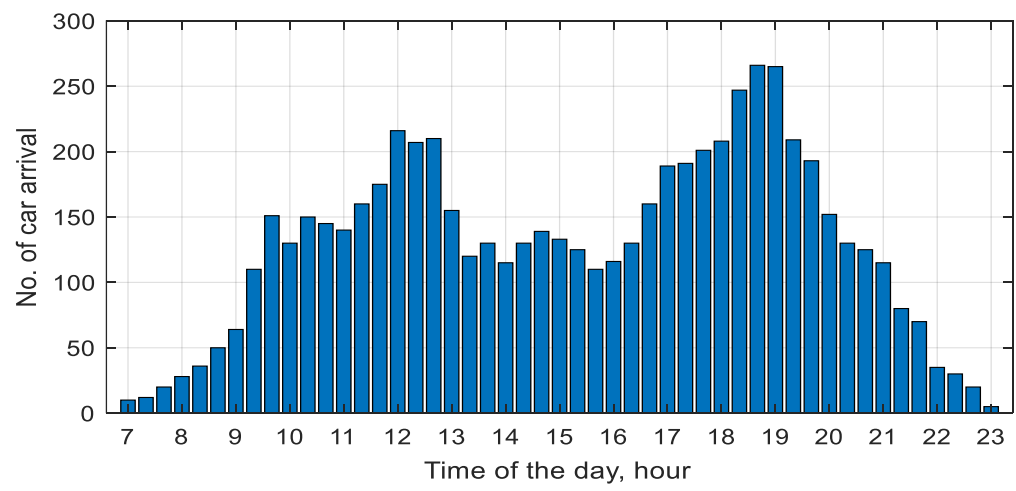

Figure 4: Average car arrival patterns over hours in a day. The parking data model for several days from 7:00 am to 11:00 pm. 

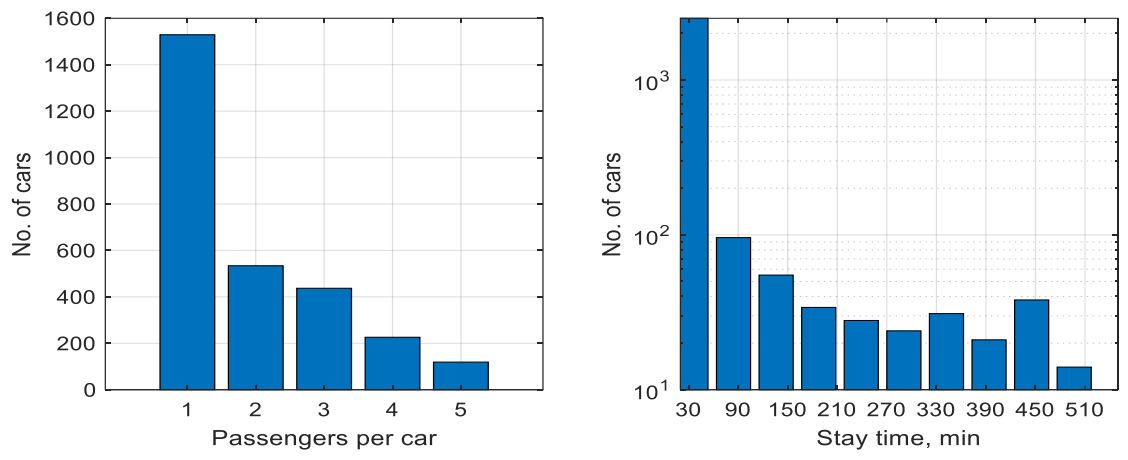

Figure 5: Histograms of Average users over a day, (a) the average number of passengers per car, and (b) the average stay time of the cars.

\section{SIMULATION RESULTS}

We consider a real parking lot as a model for implementing and evaluating the smart parking system, as illustrated in Fig. 3. The simulator is built in MATLAB and solved a nonlinear optimization problem (described in (1)) in discrete time. The maximum walking and driving distances are about $200 \mathrm{~m}$ and $400 \mathrm{~m}$, respectively, according to the parking area. The weight $w_{\mathrm{c}}$ of cost related to the comfort of user $f_{\text {comfort }}$ is normalized by the maximum walking distance and the weight $w_{e 1}$ of environmental cost $f_{\text {environment }}$ is normalized by the maximum driving distance. For simplicity, weights $w_{e 2}$ and $w_{e 3}$ are set to zero in this study. The tuning factor $\alpha$ is varied between 0 and 1 . The results of each of the previously described schemes are shown. The simulation results of different control schemes are shown in Figure 6(a), (b), (c), and (d).

In Fig. 6(a), the least-travel scheme gives the shortest driving distance, while the least-walk scheme gives the longest driving distance. The adaptive-optimal scheme has a shorter driving distance than both the optimal and uncontrolled schemes, whereas the optimal scheme has a shorter driving distance than the uncontrolled scheme. In Figure 6(b), the adaptive-optimal scheme gives the shortest walking distance, while the least-travel scheme gives the longest walking distance. The optimal scheme has a shorter walking distance than the uncontrolled scheme but is longer than the least-walk scheme. The least-walk scheme in Figure 6(c) provides the shortest distance between the car and building entry, while the least-travel scheme provides the longest distance. The adaptive-optimal scheme has a shorter distance between car and building entry than the uncontrolled scheme but is longer than the optimal scheme. The adaptive-optimal scheme in Figure 6(d) spends the least amount of time in the park, while the uncontrolled scheme spends the most. The time spent by the optimal and least-travel schemes is equal, whereas the time spent by the least-walk scheme and the uncontrolled scheme is similar. Hence, the adaptive-optimal scheme significantly improves the overall time spent in the parking lot, as well as individual walking and travel distances, compared to other schemes.

(a) Driving distance per car, $\mathrm{m}$
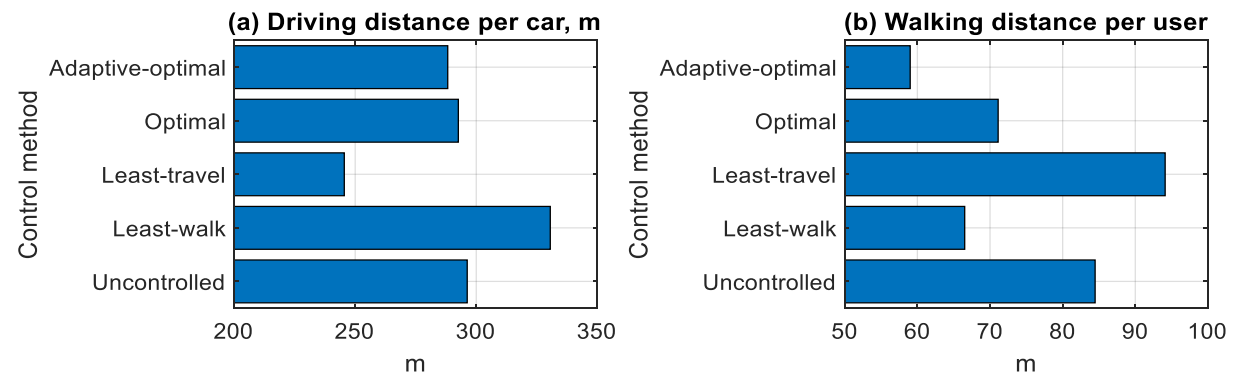

(c) Distance between car to building entrence

(d) Total time spent in park per user
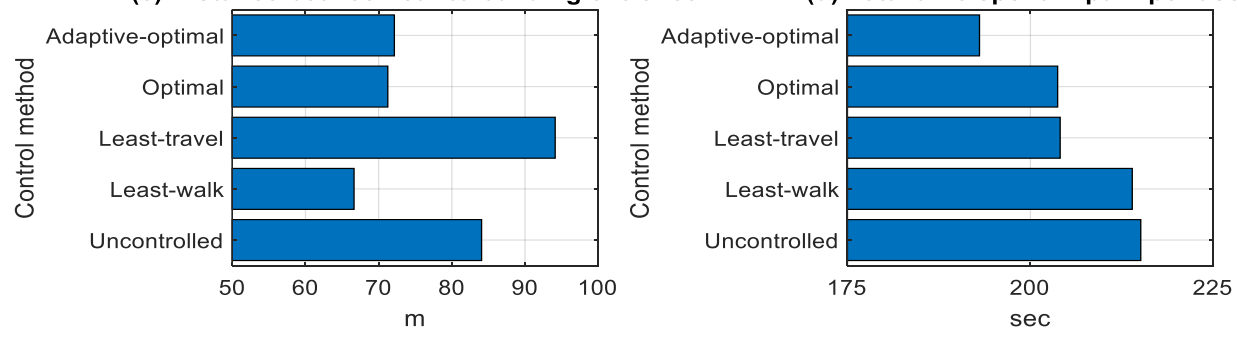

Figure 6: Comparison of the parking schemes. 


\section{CONCLUSIONS}

In this paper, we have developed an adaptive-optimal car parking management system that takes advantage of parking efficiency based on passenger data and dynamically assigns the best parking spot to each person. We assumed that the parking management system has details about the number of users, user priority, and estimated stay time when a car arrives or a parking request is made. Based on the available parking slots and the given objectives, the best parking slot is allocated. The decision-making process is fine-tuned using parking data collected over several days from a model of a large shopping complex car park, and the results are evaluated using several schemes with different objectives. The adaptive-optimal scheme is found to be successful in reducing the distance travelled and distance walked in accordance with their goals.

In the future, we will consider using machine learning to forecast usage conditions, as well as data on patterns on the roads leading up to the parking lot and data on advance reservations.

\section{ACKNOWLEDGEMENT}

This research is partly supported by Japan Society of the Promotion of Science (JSPS) Grant-in-Aid for Scientific Research (C) 20 K04531.

\section{REFERENCES}

1. Turner, B. (2011). Organization of the petroleum exporting countries (opec). The Statesmans Yearbook: The Politics, Cultures and Economies of the World 2012, 70-71.

2. Shoup, D.C. (2006). Cruising for parking. Transport policy, 13(6), 479-486.

3. Gallivan, S. (2011). IBM global parking survey: Drivers share worldwide parking woes. Technical Report, IBM, New York, NY, USA.

4. Barone, R. E., Giuffrè, T., Siniscalchi, S. M., Morgano, M. A., \& Tesoriere, G. (2013). Architecture for parking management in smart cities. IET Intelligent Transport Systems, 8(5). 445-452.

5. Bakibillah, A. S. M., Paw, Y. F., Kamal, M. A. S., Susilawati, S., \& Tan, C. P. (2021). An Incentive Based Dynamic Ride-Sharing System for Smart Cities. Smart Cities, 4(2), 532-547.

6. Handte, M., Foell, S., Wagner, S., Kortuem, G., \& Marrón, P. J. (2016). An internet-of-things enabled connected navigation system for urban bus riders. IEEE internet of things journal, 3(5), 735-744.

7. Fox, G. C., Kamburugamuve, S., \& Hartman, R. D. (2012). Architecture and measured characteristics of a cloudbased internet of things. In Proc. IEEE international conference on Collaboration Technologies and Systems (CTS), 6-12.

8. Khanna, A., \& Anand, R. (2016). IoT based smart parking system. In Proc. IEEE International Conference on Internet of Things and Applications (IOTA), 266-270.

9. Ji, Z., Ganchev, I., O'Droma, M., \& Zhang, X. (2014). A cloud-based intelligent car parking services for smart cities. In IEEE XXXIth URSI General Assembly and Scientific Symposium (URSI GASS), 1-4.

10. Choeychuen, K. (2013). Automatic parking lot mapping for available parking space detection, In Proc. IEEE 5th International Conference on Knowledge and Smart Technology (KST), 117-121.

11. Kotb, A. O., Shen, Y. C., Zhu, X., \& Huang, Y. (2015). iParker-A new smart car-parking system based on dynamic resource allocation and pricing. IEEE transactions on intelligent transportation systems, 17(9), 2637 2647.

12. Ganchev, I., O’Droma, M., \& Meere, D. (2008). Intelligent car parking locator service. International Journal ITK, 2, 166-173.

13. Hsu, C. W., Shih, M. H., Huang, H. Y., Shiue, Y. C., \& Huang, S. C. (2012). Verification of smart guiding system to search for parking space via DSRC communication. In Proc. 12th International Conference on ITS Telecommunications, 77-81.

14. Sarker, V. K., Gia, T. N., Ben Dhaou, I., \& Westerlund, T. (2020). Smart Parking System with Dynamic Pricing, Edge-Cloud Computing and LoRa. Sensors, 20(17), 4669. 